\title{
How much image noise can be added in cardiac X-ray imaging without loss in perceived image quality?
}

\author{
Amber J. Gislason-Lee*a, Asli Kumcu ${ }^{\mathrm{b}}$, Stephen M. Kengyelics ${ }^{\mathrm{a}}$, Laura A. Rhodes ${ }^{\mathrm{a}}$, \\ Andrew G. Davies ${ }^{\mathrm{a}}$ \\ ${ }^{a}$ Division of Medical Physics, Worsley Building, University of Leeds, Leeds, UK LS2 9JT \\ biMinds-IPI, Ghent University, Rectorate, Sint-Pietersnieuwstraat 25, B-9000 Ghent, Belgium
}

\begin{abstract}
Dynamic X-ray imaging systems are used for interventional cardiac procedures to treat coronary heart disease. X-ray settings are controlled automatically by specially-designed X-ray dose control mechanisms whose role is to ensure an adequate level of image quality is maintained with an acceptable radiation dose to the patient. Current commonplace dose control designs quantify image quality by performing a simple technical measurement directly from the image. However, the utility of cardiac X-ray images is in their interpretation by a cardiologist during an interventional procedure, rather than in a technical measurement. With the long term goal of devising a clinically-relevant image quality metric for an intelligent dose control system, we aim to investigate the relationship of image noise with clinical professionals' perception of dynamic image sequences.

Computer-generated noise was added, in incremental amounts, to angiograms of five different patients selected to represent the range of adult cardiac patient sizes. A two alternative forced choice staircase experiment was used to determine the amount of noise which can be added to a patient image sequences without changing image quality as perceived by clinical professionals. Twenty-five viewing sessions (five for each patient) were completed by thirteen observers. Results demonstrated scope to increase the noise of cardiac X-ray images by up to $21 \% \pm 8 \%$ before it is noticeable by clinical professionals. This indicates a potential for $21 \%$ radiation dose reduction since X-ray image noise and radiation dose are directly related; this would be beneficial to both patients and personnel.
\end{abstract}

Keywords: Image quality quantification, cardiac X-ray imaging, simulated image noise, staircase psychophysics experiment, medical image quality, perception measurement, radiation dose reduction

\section{INTRODUCTION}

In the treatment of coronary heart disease, dynamic X-ray imaging systems are used during percutaneous coronary interventional (PCI) procedures to see anatomy and clinical devices inside the human body. X-ray system settings are controlled automatically by specially-designed X-ray dose control mechanisms whose role is to ensure an adequate level of image quality is maintained with an acceptable radiation dose to the patient. Currently, commonplace dose control designs quantify image quality by performing a simple technical measurement directly from the image ${ }^{1}$. If image quality is set too high, unnecessarily high levels of X-ray dose are used.

$\mathrm{X}$-ray radiation doses from cardiac interventional procedures are the highest of any routine $\mathrm{x}$-ray examination ${ }^{2}$. Xray exposure is harmful to humans, and patients have been reported to suffer from transient and permanent skin damage caused by these cardiac procedures ${ }^{3-6}$. In addition, multiple high dose procedures being performed on relatively young patients may cause radiation-induced genetic effects and cancer later in life ${ }^{7}$. There were over 92,000 interventional cardiac procedures performed in the UK in 2012, with numbers increasing each year. As interventional procedures become longer, more complicated, and more frequent, it is increasingly important to optimize settings to minimize dose whilst maintaining adequate image quality, as enforced by the "as low as reasonably achievable" (ALARA) principle.

\footnotetext{
*a.j.gislason@leeds.ac.uk; phone +44 113343 8317; http://medhealth.leeds.ac.uk/info/523/division_of_medical_physics
} 
X-ray dose is directly related to image quality, however measuring the quality of X-ray images is not straight forward. Technical aspects of image quality, often produced by computer analysis of phantom or test object images, are reproducible and can provide excellent means of analyzing the performance of X-ray system components. Unfortunately it is not possible to translate technical measurements into the imaging of human subjects; it is not well understood how changes in technical image quality are perceived by a clinician ${ }^{8,9}$. For example, when treating a patient's heart, a clinician using a cardiac X-ray system may not notice image degradation caused by reducing the X-ray dose. The utility of cardiac patient X-ray images is in their interpretation by a cardiologist during an interventional procedure.

An intelligent dose control design would utilize a clinically-relevant measure of image quality; i.e. image perception by a cardiologist should be considered to ascertain the required level of image quality to set the dose control. With the long term goal of devising an image quality measurement for intelligent dose control, we aim to investigate the impact of changes in technical measurements on image perception. Specifically, we will determine the amount of noise which can be added to a patient image without making any difference to the perceived quality of the image. Noise is directly related to radiation dose, therefore results may demonstrate potential for a reduction in radiation dose used for PCI procedures. The impact of such a result put into practice would not only affect patient radiation dose; it would also reduce the daily exposure incurred by catheter lab personnel. In our local cardiac catheter lab, there may be 6 to 12 staff members depending on the given procedure.

\section{METHODS}

Angiograms were selected from 5 different PCI patients in our local cardiac catheter laboratory; images were made anonymous and the national research ethics committee approved their use for this study. Images were selected to represent the range of adult cardiac patient sizes (body mass index 23 to $44 \mathrm{~kg} \mathrm{~m}^{-2}$ ) and to include angular cardiac views commonly used in clinical practice. Images were acquired on an Allura Xper FD10 interventional X-ray system (Philips Healthcare, The Netherlands), modified by the manufacturer to allow for image capture without computer image enhancement processing that is normally applied to clinical images.

To avoid the unethical capture of a patient image repeatedly with varying dose levels, computer-generated quantum noise was added to patient images to simulate dose reduction. For each patient image, a collection of that image representing $1 \%$ to $99 \%$ dose reduction was created by adding corresponding increments of quantum noise - pixel by pixel, one frame at a time. An example is shown in Figure 1; on the left hand side is an image frame from the original angiogram as acquired on the X-ray system during the PCI procedure. On the right hand side is the image frame with noise added to simulate $60 \%$ dose reduction. The Philips Allura X-ray system had been used to perform calibrations and technical measurements of image quality required for the image synthesis software used to add image noise. The software had been validated using objective noise measurements and a subjective threshold contrast detail detectability (TCDD) study; a full software description is beyond the scope of this paper.

Images were viewed on a medical grade monitor with appropriately dimmed ambient lighting and placement of the monitor to simulate the cardiac catheter laboratory. Thirteen observers working in the field of medical imaging each viewed between two and four patients, depending on their time available. There were 25 observations, that is five viewing sessions for each patient. The software used to run the viewing sessions and determine the observers' perception of image degradation was written in MATLAB (The Mathworks Inc, Natick, USA) specifically for this task, executing a "staircase" or "transformed up/down" psychophysics experiment ${ }^{10}$. This experimental design was selected because it has been shown to maximize efficiency of observer perception tests such as the one required for this study ${ }^{11,12}$.

The original and degraded images of the same patient were shown side by side as an image pair in computer operated viewing sessions. The left and right placement of the images in the pair were randomized by the viewing software and recorded in a key file on the host computer. Observers were asked to focus their attention on the clarity with which the coronary arteries of the heart were shown, answering the question "which side [left or right, in the image pair] shows the vessels more clearly?" in a two alternative forced choice (2AFC) test. The level of degradation was set high (60\% dose reduction) in the first few image pairs, making the difference between the left and right images apparent; these relatively easy decisions allowed for a period of training for the observers ${ }^{10}$, and results from training images were not used for data analysis. The beginning of the staircase experiment was designed to provide the observers with time to develop confidence and become comfortable with the process. 


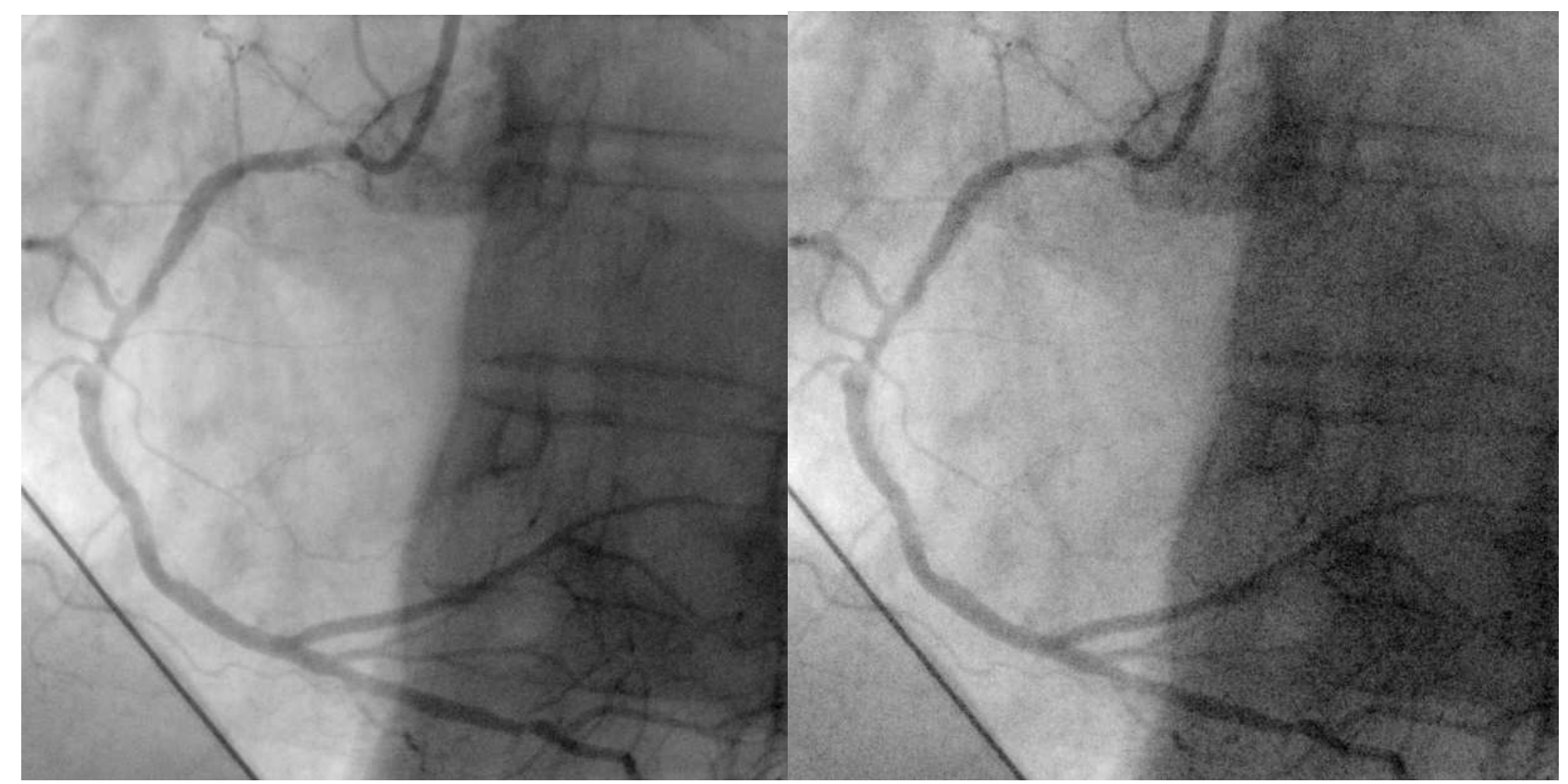

Figure 1. Single image frame from original angiogram (left) and with noise added to simulate $60 \%$ dose reduction (right).

The 1 up / 3 down rule ${ }^{12}$ was used following training: when an observer chose the original image three consecutive times (three "correct" responses, as the original had less noise), the level of degradation was reduced in the next image pair - a step down; when the observer chose the degraded image one time (an "incorrect" answer as the degraded image had more noise), the level of degradation was increased in the next image pair - a step up. A level of degradation was eventually reached where the observer had difficulty deciding between the original and degraded image, indicating that the degradation was no longer perceived. Using the still frame in Figure 1 as an example, the degradation in the right hand image will have been reduced until the left and right hand images look the same. Because it was 2AFC test i.e. the observer could not state that both images were the same, the steps then went up and down in degradation level as the observer was forced to choose either left or right. Several reversals in direction around a certain degradation level represented the observers' inability to consistently make decisions at that level. The mean of the reversal points was the estimated level of degradation no longer perceived by the observer, known as the threshold or point of subjective equality (PSE). The degree of degradation (size of step) decreased after each reversal to maximize accuracy and efficiency of the staircase experiment ${ }^{13}$. Each staircase terminated when the precision of the PSE estimate was below a threshold or the observer had viewed fifty image pairs; no time limit was imposed.

\section{RESULTS}

The mean PSE was $21 \% \pm 8 \%$ dose reduction, demonstrating that observers did not perceive dose reductions greater than $21 \%$. Two examples of staircase experimental results are shown in Figure 2. The light-colored dots represent "correct" responses and the filled in dots represent "incorrect" responses; the X's represent a reversal in direction. On both the staircases the training period was ten trials; in other cases the steps went up and then downward again as the observer's eyes became trained and confidence grew. Although the two shown staircases have differing shape, the PSEs or thresholds, shown by the dotted lines, were very similar - 19-20\% dose reduction; there were nine and eight reversals on the left and right hand side staircases respectively. 

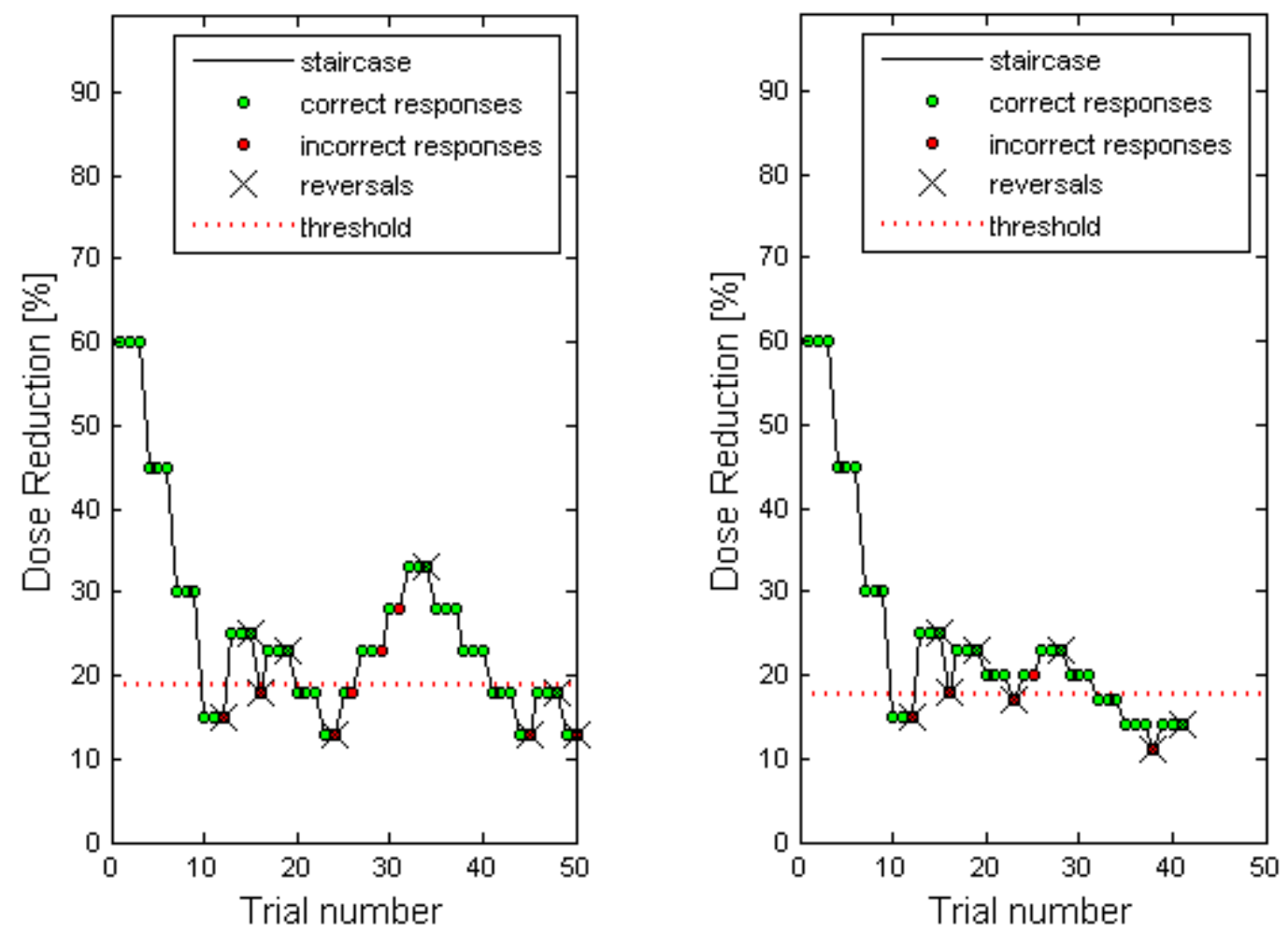

Figure 2. Staircase experimental results from two observers. The threshold denoted by the red dotted line is the point of subjective equality.

\section{DISCUSSION}

This study would not have been possible without the computer-generated dynamic clinical (human) image sequences of varying dose levels generated via the noise simulation software, which in turn required raw (unprocessed) image data. The software was created using image data from a specific image acquisition mode on the Philips Allura Xper cardiac interventional X-ray system in Leeds, in order to simulate the effect that reducing dose on this particular Xray system has on clinical images of PCI patients. For a different imaging mode or a different X-ray system, recalibration would be required using flat field image data from that mode or X-ray system, in order to accurately simulate dose reduction. In cardiac X-ray imaging, the peak tube voltage is controlled by the dose control, changing with different patient sizes and different patient projections. Therefore in order for a dose reduction simulation technique to be useful for cardiac interventional X-ray imaging, it must address the full range of peak tube voltage values which may be set by the system. The software developed in this study is the first, to the authors' knowledge, to address the full range of X-ray tube voltages used in clinical practice in cardiac X-ray imaging.

With both the noise simulation and staircase software programs implemented, a time-efficient method of measuring medical image quality perception was found. Two interleaved staircase experiments were completed in an average time of thirty minutes in a single viewing session. With settings adjusted to fit the experiment, the perception measurements were completed with high precision.

Results suggest that the method used for this study can be implemented as a clinically-relevant optimization experiment to help achieve optimal balance between image quality and radiation dose. Physicists generally utilize technical measurements of X-ray system performance or image quality from static phantom-based experiments to optimize cardiac X-ray systems ${ }^{14-16}$. However, utilizing subjective assessments of dynamic patient image sequences by 
clinicians would make optimization more clinically relevant ${ }^{8,9}$. It has been suggested, specifically for cardiac X-ray imaging, that image quality is often higher than is required for the clinical task ${ }^{17}$, causing unnecessarily high levels of radiation dose to patients and personnel; this is not in keeping with the ALARA principle.

Image enhancement normally used in the catheter labs may impact the PSE should this staircase experiment be repeated for processed images. Such future work would be required in order to implement the method used in this study as an optimization experiment, to accurately represent clinical practice. The study design may also be applied to other cardiac X-ray imaging procedures such as electrophysiology and trans-catheter aortic valve implantation.

\section{CONCLUSIONS}

A staircase psychophysics experiment was completed using dynamic clinical image sequences of varying dose levels to determine whether dose can be reduced without impacting image quality perception. Results demonstrated scope to increase noise of cardiac X-ray images by up to $21 \%$ before it is noticeable by clinical professionals, indicating a potential to reduce radiation dose by $21 \%$ during percutaneous coronary interventional procedures.

\section{ACKNOWLEDGEMENTS}

This work was funded by Philips Healthcare, NL. Part of this work has been performed in the project PANORAMA, cofunded by grants from Belgium, Italy, France, the Netherlands, and the United Kingdom, and the ENIAC Joint Undertaking.

\section{REFERENCES}

[1] Gislason, A.J., Hoornaert, B., Davies, A.G. and Cowen, A.R., “Allura Xper Cardiac System Implementation of Automatic Dose Rate Control," Philips Healthcare (2011).

[2] Chida, K., Kagaya, Y., Saito, H., Ischibashi, T., Takahashi, S. and Zuguchi, M., "Evaluation of patient radiation dose during cardiac interventional procedures: What is the most effective method?" Acta Radiol. 50, 474-481 (2009).

[3] Vlietstra, R.E., Wagner, L.K., Koenig, T. and Mettler, F., "Radiation burns as a severe complication of fluoroscopy guided cardiological interventions," Journal of Interventional Cardiology 17, 131-142 (2004).

[4] Frazier, T.H., Richardson, J.B., Fabre, V.C. and Callen, J.P., "Fluoroscopy-induced chronic radiation skin injury: A disease perhaps often overlooked," Arch. Dermatology 143, 637-640 (2007).

[5] Henry, M.F., Maender, J.L., Shen, Y., Tschen, J.A., Subrt, P, Schmidt, J.D. and Hsu, S., "Fluoroscopy-induced chronic radiation dermatitis: A report of three cases," Dermatology Online J. 15, 1-5 (2009).

[6] Balter, S., Hopewell, J.W., Miller, D.L., Wagner, L.K. and Zelefsky, M.J., "Fluoroscopically guided interventional procedures: A review of radiation effects on patients' skin and hair," Radiology 254, 326-341 (2010).

[7] ICRP, "Publication 103: Recommendations of the International Commission on Radiological Protection," Ann. ICRP (2007).

[8] Tapiovaara, M.J., "Review of relationships between physical measurements and user evaluation of image quality." Radiation protection dosimetry 129, 244-8 (2008).

[9] Båth, M., "Evaluating imaging systems: practical applications," Radiation protection dosimetry. 139, 26-36 (2010).

[10] Kingdom, F.A.A. and Prins, N., [Psychophysics: A practical introduction], Elsevier, London (2010).

[11] Treutwein, B., "Minireview: Adaptive Psychophysical Procedures," Vision Research 35, 2503-2522 (1995).

[12]Levitt, H., "Transformed Up-Down Methods in Psychoacoustics," The Journal of Acoustical Society of America, 467-477 (1970).

[13] Garcia-Perez, M.A., "Yes-No Staircases with Fixed Step Sizes: Psychometric Properties and Optimal Setup," Optometry and Vision Science 78, 56-64 (2001). 
[14] Tapiovaara, M.J., Sandborg, M. and Dance, D.R., "A search for improved technique factors in paediatric fluoroscopy," Physics in Medicine and Biology 44, 537-559 (1999).

[15] Gislason, A.J., Davies, A.G. and Cowen, A.R., "Dose optimization in pediatric cardiac x-ray imaging," Medical Physics 37, 5258-5269 (2010).

[16] Gislason-Lee, A.J., McMillan, C., Cowen, A.R. and Davies, A.G., "Dose optimization in cardiac x-ray imaging," Medical Physics 40, 091911-1091911-11 (2013).

[17]Dixon, R.G. and Wagner, L., "Managing Image Quality and Patient Dose in the Angiography Suite: Do you Really Need That Image Quality?” Techniques in Vascular and Interventional Radiology, 13, 183-187 (2010). 